\title{
BMJ Open Educational and training interventions aimed at healthcare workers in the detection and management of people with mental health conditions in South and Southeast Asia: systematic review protocol
}

\author{
Kamrun Nahar Koly (D) , ${ }^{1}$ Cleo Baskin (D) , ${ }^{2}$ Ivy Lata (D) , Mala Rao, ${ }^{2}$ \\ Sabrina Rasheed, ${ }^{1}$ Graham Law, ${ }^{3}$ Shamini Gnani (i) ${ }^{2}$
}

To cite: Koly KN, Baskin C, Lata I, et al. Educational and training interventions aimed at healthcare workers in the detection and management of people with mental health conditions in South and Southeast Asia: systematic review protocol. BMJ Open 2021;11:e045615. doi:10.1136/ bmjopen-2020-045615

- Prepublication history and supplemental material for this paper is available online. To view these files, please visit the journal online (http://dx.doi. org/10.1136/bmjopen-2020045615).

Received 08 0ctober 2020 Accepted 07 June 2021

Check for updates

(C) Author(s) (or their employer(s)) 2021. Re-use permitted under CC BY-NC. No commercial re-use. See rights and permissions. Published by BMJ.

For numbered affiliations see end of article.

Correspondence to Dr Kamrun Nahar Koly; koly@icddrb.org

\section{ABSTRACT}

Introduction The mental health burden and treatment gap in South and Southeast Asia is high and significant. Capacity building of healthcare workers is essential to support programmes related to the detection and management of patients with mental health conditions. We aim to conduct a systematic review to summarise the research on educational, training and capacity-building interventions aimed at the healthcare workforce in detection and management of mental health conditions in South and Southeast Asia.

Objective To synthesise evidence on (1) the types of educational and training interventions that have been used to improve the knowledge, skills and attitudes of healthcare workers in South and Southeast Asian countries in the detection and management of mental health conditions; (2) the effectiveness, including cost-effectiveness of the interventions; and (3) the enabling factors and barriers that influence the effectiveness of these interventions.

Methods and analysis This review will be conducted in accordance with Preferred Reporting Items for Systematic Reviews and Meta-Analyses Extension for Scoping Reviews guidelines. We will search six electronic databases: MEDLINE, EMBASE, PsycINF0, Cochrane Library, Cumulative Index to Nursing and Allied Health Literature, and Global Health for empirical studies published from 1 January 2000 to 31 August 2020. Search results from each database will be combined and uploaded in Covidence library. Title, abstract and full-text screening, and data extraction of each included study will be performed by two independent reviewers. Disagreements between reviewers will be resolved by a third reviewer and study team. Quality of included studies will be assessed by the modified Cochrane Collaboration tool and ROBINS-I tool. Data will be synthesised and if a metaanalysis is not appropriate, a stepwise thematic analysis will be performed.

Ethics and dissemination Ethics approval is not required for this study. Findings will be disseminated through peer-reviewed publications, fact sheets, multimedia press briefings, conferences, seminars and symposia. PROSPERO registration number CRD42020203955.

\section{Strengths and limitations of this study}

- This is the first systematic review of evidence of the effectiveness of educational and training interventions for healthcare workers to improve the detection and management of mental health disorders in the context of South and Southeast Asia which will assist policymakers to strengthen human resources and the mental healthcare system in the included countries.

- This research protocol follows the Preferred Reporting Items for Systematic Reviews and MetaAnalyses Extension for Scoping Reviews protocol to minimise and protect against risk of biases and allow peer review.

- There may be inadequate numbers of published studies in this area limiting the ability to undertake a meta-analysis.

- This review excludes published studies that are not in English and may limit the findings by introducing a language bias.

\section{BACKGROUND}

Effective health systems should deliver adequate and comprehensive health services, including mental health services to all people. ${ }^{1}$ However, health systems globally and particularly in low/middle-income countries (LMICs) have inadequately responded to the high burden of mental health disorders. ${ }^{1}$ More than $70 \%$ of people in LMICs experience a mental health disorder ${ }^{23}$ and this burden is more extensive in South and Southeast Asian countries. ${ }^{45}$ The disability-adjusted life year for mental illness in South Asian countries is 2000 per 100000 population $^{6}$ and 11000 in Southeast Asian countries. ${ }^{78}$ In these regions, the 
prevalence of mental health disorders varies among countries and reported to range from $6.5 \%$ to $31.0 \% .^{8-10}$ Despite the high burden, the mental health treatment gap in LMICs is very high with between $76 \%$ and $84 \%$ of people with a mental illness not receiving treatment. ${ }^{11}$ The impact of untreated mental disorders on individuals and their families often leads to low quality of life, stigma, discrimination, poor physical health and premature mortality. ${ }^{12}$

A trained health workforce is one of the key components of health systems, but there is scarcity and inequity in distribution and inefficiency of health human resources in developing countries. ${ }^{13-15}$ These problems are more acute for mental health. ${ }^{13-15}$ In South Asian countries, per 100000 people the number of psychiatrists range from 0.3 in India to 2.4 in Maldives and 0.2 in other South Asian countries compared with 10.5 in the USA, which shows gross inadequacy. ${ }^{16} 17$ In addition, the mental health workforce is mainly concentrated in urban areas, where there are few primary healthcare workers trained in mental healthcare. Inadequate mental health training among primary healthcare workers and inadequate staffing of mental health specialists, together with a lack of appropriate skill mix, affect the overall care pathways in terms of diagnosis, treatment and prevention of mental illness. ${ }^{13} 18$

Human resources are an integral but a costly component of all health systems. ${ }^{19}$ Researchers have reported that training programmes on mental health are effective in enhancing knowledge, attitudes and practices among the healthcare professionals. ${ }^{20}$ Task sharing or a collaborative stepped care approach is one of the arrangements in which non-clinical specialists, like lay workers or informal caregivers, receive training and appropriate supervision by the specialist health professionals to enable them to screen and manage a mental disorder. ${ }^{21}$ Due to the workforce shortage in low-resource settings in South Asia, adopting these approaches may help to address some of the unmet mental healthcare needs. ${ }^{22} 23$

In several studies in LMICs, researchers also have reported that these care methods led by lay health workers were successful in managing depression and anxiety in primary care setting. ${ }^{24-26}$ This is an important finding as most primary care settings in LMICs have no regular specialists. In order to make sustainable and effective changes to practice among trained healthcare workers, ongoing learning and skill development needs to be integral to the capacity-building intervention. ${ }^{27}$ Scaling up a stepped care model within the health systems might offer opportunities related to service provision. ${ }^{28}$ There might be other interventions that are suitable for LMIC settings; however, there is currently no systematic review on the topic of interventions related to building capacity of the healthcare workforce. This review is designed to synthesise evidence about best practices from South Asian and Southeast Asian countries to strengthen mental healthcare pathways.

\section{AIMS AND OBJECTIVES}

Our aim for this systematic review is to synthesise evidence about the effectiveness of interventions aimed at developing the healthcare workforce to strengthen mental healthcare in South Asian and Southeast Asian countries.

Our objectives are to determine: (1) the types of educational and training interventions that have been used to improve the knowledge, skills and attitudes of healthcare workers in South and Southeast Asian countries in the detection and management of mental health conditions, (2) the effectiveness including cost-effectiveness of these interventions; and (3) the enabling factors and barriers that influence the effectiveness of these interventions.

\section{METHODS AND ANALYSIS}

This current protocol has been drafted following the recommendations by the standard guideline Preferred Reporting Items for Systematic Reviews and Meta-Analyses (PRISMA) Protocol checklist ${ }^{29}$ (see online supplemental file 1). We will also conduct and report the findings of this systematic review according to PRISMA guidelines upon completion. This review has also been registered in the International Prospective Register of Systematic Reviews (PROSPERO, https://www.crd.york.ac.uk/PROSPERO).

\section{Inclusion and exclusion criteria}

\section{Types of participants}

We will include studies conducted among healthcare workers who are directly involved in the provision of clinical care. Healthcare workers include doctors, nurses, primary healthcare workers, community workers, community volunteers such as Shastha Shebika and lay health counsellors. Volunteers or medical or nursing students will be excluded as study participants because they do not reflect the formal healthcare system and are not directly involved in the provision of clinical care.

\section{Types of interventions}

Interventions of interest include any activity associated with education, training or capacity building that aims to improve the ability of healthcare workers to detect and manage mental health disorders. The training can take place in any setting, face to face or online, and over any duration. We will exclude studies where programmes other than educational and training interventions were used.

\section{Types of outcome measures}

We will include studies that report improvements in detection and management of mental health conditions by healthcare workers, and their knowledge, skills and attitudes of mental health conditions. We will include both common and severe mental health disorders, as defined by the WHO's International Classification of Diseases (ICD-10) and ICD-II or Diagnostic and Statistical Manual of Mental Disorders (DSM-IV) and DSM-V. We will also include case studies with more descriptive outcomes such 
as the number of healthcare workers trained and the number of the people detected with mental illness. We will exclude studies with outcomes that do not include mental health conditions.

Types of studies

We will include all available studies such as randomised controlled trials, quasi-randomised controlled trials, propensity matched trials, non-randomised controlled trials, pre-intervention and post-intervention studies, and observational studies such as case reports, case studies, systematic reviews and qualitative studies. We will exclude publications that are conference proceedings, posters or books, and book chapters and studies whose full text were unavailable.

\section{Comparator}

We will include all studies regardless if they had a comparator group or not. If a comparator group is present, then they will be included if they were provided any intervention or usual training or if they were provided with no intervention.

\section{Study setting}

We will include studies that were conducted in all healthcare settings, such as primary healthcare centres, community care centres, public and/or private hospitals, or clinics. All studies have to take place in South Asian and Southeast Asian countries. We will exclude studies where interventions were provided outside healthcare settings.

\section{Information sources}

We will search the following electronic databases to retrieve relevant articles: MEDLINE, EMBASE, PsycINFO, Cochrane Library, Cumulative Index to Nursing and Allied Health Literature (CINAHL), and Global Health.

\section{Study records}

We will use reference management software Covidence, an online systematic review tool recommended by the Cochrane Collaboration, to organise articles retrieved from the comprehensive literature search from different electronic databases. ${ }^{30}$

\section{Context}

We will include studies conducted in the South Asia and Southeast Asia region. South Asian countries are defined by the World Bank and Southeast Asia region, defined by WHO, to include the following countries: Bangladesh, India, Pakistan, Sri Lanka, Afghanistan, Nepal, Bhutan, Maldives, Democratic People's Republic of Korea, Indonesia, Myanmar, Thailand and TimorLeste. $^{31}{ }^{32}$ We will exclude studies conducted outside South Asia and Southeast Asia.

\section{Study period}

We will include studies published from 1 January 2000 to 31 August 2020.

\section{Study language}

We will include full-text studies published in the English language and exclude full-text non-English-language publications.

\section{Search strategy}

The study team will conduct a systematic review of educational and training interventions aimed at healthcare workers in the detection and management of people with mental health conditions in South Asia and Southeast Asia.

A comprehensive search strategy will be developed, which will involve mapping of concepts, and for each database the search terms will be adapted. We will develop the concepts for our key search terms using the population, intervention, comparison, outcome framework in table 1 . Please see the final search strategy of the databases MEDLINE, EMBASE, PsycINFO, Global health, CINAHL and Cochrane in online supplemental file 2.

\section{Screening and data extraction}

Search results from different electronic databases will be combined and uploaded in a single Covidence library. Duplicate articles will be removed. A data extraction framework will be designed and implemented for studies eligible for inclusion in this review. Studies retrieved using the search strategy will be independently screened by title and abstract by two reviewers. Where the two reviewers agree about inclusion of a study, this study will be put forward for full-text screening. Two reviewers will upload the available articles in the Covidence and screen full text of retrieved articles independently. In case of disagreement, the study will be independently reviewed by a third researcher. Data will be extracted from studies finally selected for inclusion.

Table 1 Key terms used for developing comprehensive search strategy

\begin{tabular}{llll}
\hline Population & Intervention & Comparison & Outcome \\
\hline $\begin{array}{l}\text { Healthcare workers in South } \\
\text { Asia and Southeast Asia }\end{array}$ & $\begin{array}{l}\text { Training, education and } \\
\text { capacity building }\end{array}$ & $\begin{array}{l}\text { Control group with } \\
\text { any intervention or } \\
\text { usual training or no }\end{array}$ & $\begin{array}{l}\text { Improvement in detection and } \\
\text { management of mental health } \\
\text { conditions by healthcare workers }\end{array}$ \\
& & interventions & Improvement of knowledge, skills \\
& & and attitudes of the mental health \\
& & conditions by healthcare workers
\end{tabular}




\section{Quality assessment}

Quality of the study or risk of bias will be assessed by two independent reviewers. All randomised and quasirandomised controlled trials will be assessed using the modified Cochrane Collaboration tool where bias is assessed as a judgement of high, low, unclear across five domains (selection, performance, attrition, reporting and other). For non-randomised studies of interventions, the ROBINS-I tool will be used to assess risk of bias. Any disagreements between the two researchers on the quality assessment will be resolved by discussion with the study review team.

\section{Descriptive analysis and meta-analysis}

The review synthesis will be structured around the type of intervention in relationship to detection and management, country and population characteristics such as the category of healthcare worker and other sociodemographic information, mental health condition, and age range. We will provide summaries of intervention effects where possible, calculate mean differences or percentages for continuous outcomes. We will pool data for dichotomous and continuous outcomes and calculate risk ratios/ prevalence and 95\% CIs for dichotomous outcomes.

We anticipate substantial variation among studies that meet our inclusion criteria. If possible, we will visually explore any heterogeneity in results for primary outcomes using bubble plots or box plots (displaying medians, IQRs and ranges). If there are sufficient data, we will also explore heterogeneity in findings for primary outcomes using meta-regression. For data analysis, Stata V.16 will be used. Where data are not available, we will provide a narrative synthesis of the findings from the included studies.

\section{Patient and public involvement}

The patients and public will not be involved in this review.

\section{ETHICS AND DISSEMINATION}

No ethical approval is required for this review as data have already been published. Findings of this systematic review will be presented for peer review in an appropriate journal and presented to researchers and clinicians at suitable conferences. Findings will be disseminated through peer-reviewed publications, fact sheets and multimedia press briefings, for example, in conferences or seminars or symposia.

\section{AMENDMENTS}

If this protocol needs to be amended, the date of each amendment, changes and rationale will be described in this section, and updated on PROSPERO registration.

\section{Author affiliations}

${ }^{1}$ Health System and Population Studies Division, International Centre for Diarrhoeal Disease Research Bangladesh, Dhaka, Bangladesh
${ }^{2}$ Department of Primary Care and Public Health, Imperial College London, London, UK

${ }^{3}$ School of Health and Social Care, University of Lincoln, Lincoln, UK

Twitter Mala Rao @RaoMala

Acknowledgements The authors would like to thank the medical librarian and other support staff of Imperial College London and International Centre for Diarrhoeal Disease Research, Bangladesh for their assistance in developing the search strategy for this review. icddr,b is grateful to the governments of Bangladesh, Canada, Sweden and the UK for providing unrestricted support.

Contributors KNK, CB, IL and SG developed the study protocol with contributions from MR, GL and SR. KNK, CB and IL drafted the initial manuscript from the systematic review protocol submitted for registration to PROSPERO. KNK, CB, IL and SG developed the search strategy with the support of the librarian from Imperial College London. KNK and CB will screen the title, abstract, full texts of the retrieved articles; and SG will resolve any conflict as the third reviewer in discussion with other members of the research team to seek agreement. KNK, CB and IL will conduct data extraction. All authors contributed to generate the idea and conception of the systematic review. All authors will critically read, provide feedback and revise the manuscript, and approve the final version of the manuscript for submission.

Funding UK Research and Innovation Global Challenges Research Fund awarded to Imperial College London, UK (award or grant number: P84624) to conduct this work. No other specific grant from any funding agency in the commercial, public or not-for-profit sectors was received for this research.

Competing interests None declared.

Patient and public involvement statement No patient involved

Patient consent for publication Not required.

Ethics approval No ethical approval is required for this review as data have already been published.

Provenance and peer review Not commissioned; externally peer reviewed.

Supplemental material This content has been supplied by the author(s). It has not been vetted by BMJ Publishing Group Limited (BMJ) and may not have been peer-reviewed. Any opinions or recommendations discussed are solely those of the author(s) and are not endorsed by BMJ. BMJ disclaims all liability and responsibility arising from any reliance placed on the content. Where the content includes any translated material, BMJ does not warrant the accuracy and reliability of the translations (including but not limited to local regulations, clinical guidelines, terminology, drug names and drug dosages), and is not responsible for any error and/or omissions arising from translation and adaptation or otherwise.

Open access This is an open access article distributed in accordance with the Creative Commons Attribution Non Commercial (CC BY-NC 4.0) license, which permits others to distribute, remix, adapt, build upon this work non-commercially, and license their derivative works on different terms, provided the original work is properly cited, appropriate credit is given, any changes made indicated, and the use is non-commercial. See: http://creativecommons.org/licenses/by-nc/4.0/.

\section{ORCID iDs}

Kamrun Nahar Koly http://orcid.org/0000-0003-2466-8139

Cleo Baskin http://orcid.org/0000-0001-6254-8707

Ivy Lata http://orcid.org/0000-0002-4733-939X

Shamini Gnani http://orcid.org/0000-0001-6246-9590

\section{REFERENCES}

1 Semrau M, Evans-Lacko S, Alem A, et al. Strengthening mental health systems in low- and middle-income countries: the Emerald programme. BMC Med 2015;13:79.

2 Alloh F, Regmi P, Hemingway A. Increasing suicide rates in Nigeria. Afr Health J 2018.

3 Rathod S, Pinninti N, Irfan M, et al. Mental health service provision in low- and middle-income countries. Health Serv Insights 2017;10:117863291769435.

4 Sharan P, Sagar R, Kumar S. Mental health policies in southeast Asia and the public health role of screening instruments for depression. WHO South East Asia J Public Health 2017;6:5.

5 Ranjan JK, Asthana HS. Prevalence of mental disorders in India and other South Asian countries. Asian J Epidemiol 2017;10:45-53. 
6 Whiteford HA, Ferrari AJ, Degenhardt L, et al. The global burden of mental, neurological and substance use disorders: an analysis from the global burden of disease study 2010. PLoS One 2015;10:e0116820.

7 Reddy MS. Depression: the disorder and the burden. Indian $J$ Psychol Med 2010;32:1-2.

8 India State-Level Disease Burden Initiative Mental Disorders Collaborators. The burden of mental disorders across the states of India: the global burden of disease study 1990-2017. Lancet Psychiatry 2020;7:148-61.

9 Ansari I. Mental health Pakistan: optimizing brains. Int J Emerg Ment Health 2015;17:228.

10 Hossain MD, Ahmed HU, Chowdhury WA, et al. Mental disorders in Bangladesh: a systematic review. BMC Psychiatry 2014;14:216.

11 Lund C, Tomlinson M, De Silva M, et al. Prime: a programme to reduce the treatment gap for mental disorders in five low- and middle-income countries. PLoS Med 2012;9:e1001359.

12 Ngui EM, Khasakhala L, Ndetei D, et al. Mental disorders, health inequalities and ethics: a global perspective. Int Rev Psychiatry 2010;22:235-44.

13 Ayano G. Significance of mental health legislation for successful primary care for mental health and community mental health services: a review. Afr J Prim Health Care Fam Med 2018:10:1-4.

14 Trivedi JK, Goel D, Kallivayalil RA, et al. Regional cooperation in South Asia in the field of mental health. World Psychiatry 2007;6:57.

15 Thara R, Padmavati R. Community mental health care in South Asia. World Psychiatry 2013;12:176-7.

16 Organization WH. Global health observatory data Repository, 2016. Available: http://apps.who.int/gho/data/node.main.A865

17 Ayano G. Primary mental health care services in Ethiopia: experiences, opportunities and challenges from East African country. $J$ Neuropsychopharmacol Ment Health 2016;1:113.

18 Krishnan AKI, Parvathy N, Mohan R, et al. Evidence on mental health policy gaps in South East Asia: a systematic review of South East Asian countries with special focus on Myanmar, 2020.

19 Dussault G, Dubois C-A. Human resources for health policies: a critical component in health policies. Hum Resour Health 2003;1:1.
20 Jacob KS, Sharan P, Mirza I, et al. Mental health systems in countries: where are we now? Lancet 2007;370:1061-77.

21 Fulton BD, Scheffler RM, Sparkes SP, et al. Health workforce skil mix and task shifting in low income countries: a review of recent evidence. Hum Resour Health 2011;9:1-11.

22 Abayneh S, Lempp H, Alem A, et al. Service user involvement in mental health system strengthening in a rural African setting: qualitative study. BMC Psychiatry 2017;17:1-14.

23 Patel V, Weiss HA, Chowdhary N, et al. Lay health worker led intervention for depressive and anxiety disorders in India: impact on clinical and disability outcomes over 12 months. Br J Psychiatry 2011;199:459-66.

24 Hoeft TJ, Fortney JC, Patel V, et al. Task-sharing approaches to improve mental health care in rural and other low-resource settings: a systematic review. J Rural Health 2018;34:48-62.

25 Araya R, Flynn T, Rojas G, et al. Cost-effectiveness of a primary care treatment program for depression in low-income women in Santiago, Chile. Am J Psychiatry 2006;163:1379-87.

26 Patel V, Weiss HA, Chowdhary N, et al. Effectiveness of an intervention led by lay health counsellors for depressive and anxiety disorders in primary care in Goa, India (MANAS): a cluster randomised controlled trial. Lancet 2010;376:2086-95

27 Karim MR, Huda KN, Khan RS. Significance of training and post training evaluation for employee effectiveness: An empirical study on Sainsbury's Supermarket Ltd, UK. IJBM 2012;7:141.

28 Dodd R, Palagyi A, Jan S, et al. Organisation of primary health care systems in low- and middle-income countries: review of evidence on what works and why in the Asia-Pacific region. BMJ Glob Health 2019;4:e001487.

29 Moher D, Shamseer L, Clarke M, et al. Preferred reporting items for systematic review and meta-analysis protocols (PRISMA-P) 2015 statement. Syst Rev 2015;4:1.

30 Library QU. Systematic review and other syntheses, 2017. Available: https://guides.library.queensu.ca/knowledge-syntheses

31 WHO. Who in south-east Asia, 2020. Available: http://origin.searo. who.int/about/history/en/

32 Bank W. South Asia, 2020. Available: https://data.worldbank.org/ country/8S 